\title{
Low cost, simplified gamete uterine transfer (GUT) for tubal occlusion in low resource settings: A review and a guideline
}

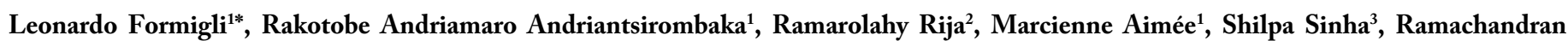
Krishnamoorthy ${ }^{1}$, Hanitra Rasoarimanana ${ }^{1}$, Nargis Ashurova ${ }^{4}$, Ella Ekogha Nsa Mve Rose ${ }^{5}$ and Graziella Badulli ${ }^{1 *}$

${ }^{1}$ CEFERMAD (Centre de Fertilité de Madagascar) Lot VK 25 bis, Ambohimanoro, Antananarivo 101, Madagascar

${ }^{2}$ Service de Réanimation Chirurgicale-Hôpital Universitaire Ravoahangy, Antananarivo, Madagascar

${ }^{3}$ Department of Obstetrics and Gynecology, Wellkin Hospital, Mauritius

${ }^{4}$ Levy Mwanawasa University Teaching Hospital, Lusaka, Zambia

${ }^{5}$ Centre de Fertilité Ibekelia Marie, Libreville, Gabon

\begin{abstract}
It has been estimated that in the world there are approximately 90 milion infertile couples, the majority of which are poor and cannot afford expensive assisted reproductive treatments (ART). The creation of centres and the use of increasingly sophisticated treatments such as intra cytoplasmic sperm injection (ICSI), vitrification, preimplantation genetic diagnosis (PGD) and so on, have created in physicians the opinion that infertility treatment can now only be accomplished in sophisticated centres and is out of reach of their capabilities and skills. Simplified intra uterine insemination (SIUI), for doctors not particularly trained in ART and not needing the use of any media, has already been described and published. However, SIUI has a major disadvantage: it may only be applied in cases with normal tubes, whereas one of the major causes of infertility in developing, poor countries is occlusion of the fallopian tubes. Gametes uterine transfer (GUT) depicted in this paper is an old technique used in the eighties and nineties and it is meant for bilateral tubal occlusion, albeit it may be used for many other infertility indications. A simple table, one and half meter long, half a meter wide with two cheap microscopes and a small benchtop centrifuge in the doctor's office together with a portable ultrasound machine with a vaginal probe is all what is needed. Oocytes are aspirated from the follicles and immediately transferred to the uterus together with the sperm. This technique has a rather low success potential; however, it may be repeated almost every month, because of its very low cost and low traumaticity similarly to IUI. The cumulative pregnancy rate after 3 or 4 attempts may reach the success rate of a conventional single attempt of IVF. Total fee for each GUT procedure has been estimated in little more than 100 USD and even after multiple GUT attempts the cumulative cost would be considerably less than a single IVF procedure and therefore much more affordable by the poor.
\end{abstract}

\section{Introduction}

The World Health Organisation (WHO) has long sustained that reproduction is an inherent right of each person, poor or rich. On the other hand, at the beginning of the IVF era in mid-seventies, and during the few years thereafter, the first doctors were less business oriented as compared to the today's situation, since the business potential of the new ART techniques had not been fully appreciated at the time. The prices of ART techniques have risen in a significant way in the last two decades, thereby increasing the gap between those patients who can afford to pay and the great majority who cannot.

Many authors have dealt with the issue of treating infertility with simplified in vitro fertilization (IVF) [1] and intra uterine insemination (SIUI) [2] in low resource settings. Some have produced special culture test tubes, not needing a $\mathrm{CO}_{2}$ incubator, where fertilisation and the first cleavage divisions take place $[3,4]$. The normal $\mathrm{pH}$ is maintained by a chemical reaction occuring in the cap of these test tubes; others insert the gametes into a small capped test tube and then insert the test tube into the patient's vagina [5]: in this last case a $\mathrm{CO}_{2}$ incubator is required to pre-equilibrate the medium $\mathrm{pH}$. In both cases however, one needs special, commercial, expensive culture media and trained staff in embryology since embryos must be searched for and identified in the test tubes at the end of the culture, evaluated and transferred into the uterus. This paper chose to confront the issue of a simplified ART with a practical approach, a sort of guideline for physicians. So far, infertility treatment is being mostly delivered in infertility centres, where only infertile patients of the middle or high class can afford expensive treatments. In our view, gynaecologists or even general practitioners (gynaecologists are not very numerous in developing countries) should be able to help their infertile patients in their own office, as they do for many ailments and diseases, without referring them to specialised centres, unless they are confronted with exceptional, specific cases. After all, many doctors already deliver simple procedures in their offices, such as intra uterine insemination (IUI), dilation and curettage (D\&C), intra uterine devices introduction, cervical diathermy, endometrial biopsies, cervical biopsies, removal of polyps, ovarian cysts transvaginal

${ }^{\star}$ Correspondence to: Graziella Badulli, CEFERMAD (Centre de Fertilité de Madagascar) Lot VK 25 bis, Ambohimanoro, Antananarivo 101, Madagascar, E-mail: graziellaparda@yahoo.it

Key words: gamete uterine transfer, GUT, low cost IVF, simplified IVF, tubal occlusion, ART, poor resource settings

Received: June 18, 2019; Accepted: June 27, 2019; Published: June 29, 2019 
aspiration and so on. This paper reproposes GUT, an outdated, simple, low cost technique, which, as far as we know, is not being used any more, because of the improvement of the IVF technique. The GUT procedure however may still be used for the treatment of one of the commonest reasons for infertility in developing countries, blocked fallopian tubes. GUT may also be used for many other causes of infertility except severe male infertility and hormonal problems. The described technique has been simplified, does not need commercial media, $\mathrm{CO}_{2}$ incubators or trained biologists and may be performed in a doctor's office, by the doctor alone, or with the help of a nurse, with acceptable results and a considerable reduction of costs, which make this technique affordable for poor couples in low resource settings. However, a few words should first be dedicated to the preliminary approach to infertility and tests, in order to reduce here too the expenditures for the couple. First, analyze whether an expectant management of a few months or even a year or two may be beneficial. Many pegnancies occur spontaneously after some time of regular intercourses. Second, limit the number of infertility investigations and tests to the bare minimum: a sperm test, a hysterosalpingography and in case of menstrual irregularities, hormonal evaluations.

\section{Gametes uterine transfer (GUT)}

This technique has been used since the early eighties by several authors [6-14] with varying results, and an average pregnancy rate of approximately $21 \%$ per attempt (Table 1 ).

GUT is not an in vitro technique; it is an in vivo technique, since fertilisation takes place inside the body, in the uterine cavity. Therefore it may be chosen also by couples who refrain from in vitro fertilisation (IVF) for ethical or religious reasons. The doctor only needs to learn how to identify Cumulus-Oocyte Complexes (COC)s in the follicular fluid, which needs a very cheap stereo microscope with a LED light coming from below. This simple lab procedure may be learnt in a few minutes. In addition, the doctor should be able to perform transvaginal ultrasound guided follicular aspiration. At the beginning, an inexperienced doctor should start by aspirating large ovarian cysts and then follicular aspiration will appear very simple and easy. Of course, there is a learning curve, as for any other procedure. GUT may be performed without anaesthesia because of the low number of follicles produced by Clomiphene ovarian stimulation.

As a medium, the patient's own serum may be used in order to reduce costs and avoid expensive media. There is no need of $\mathrm{CO}_{2}$

Table 1. Published reports of the GUT technique and results

\begin{tabular}{|c|c|c|c|c|}
\hline Report & $\begin{array}{c}\text { Year of } \\
\text { publication }\end{array}$ & $\begin{array}{c}\text { Ovarian } \\
\text { stimulation }\end{array}$ & $\begin{array}{c}\text { Number of } \\
\text { patients }\end{array}$ & $\begin{array}{l}\text { Pregnancies } \\
\text { (\%) }\end{array}$ \\
\hline Craft et al. [6] & 1982 & $\mathrm{CC}^{*}$ & 31 & $2(6 \%)$ \\
\hline Veersema et al. [7] & 1989 & $\mathrm{CC}^{*}$ & 6 & $2(33 \%)$ \\
\hline Kubik et al. [8] & 1990 & U HMG** & 20 & $3(15 \%)$ \\
\hline Rizk et al. [9] & 1990 & $\begin{array}{l}\mathrm{CC}^{*}+\mathrm{U} \\
\mathrm{HMG}^{* *}\end{array}$ & 20 & $4(20 \%)$ \\
\hline Sterzik et al. [10] & 1991 & -- & 9 & $4(44 \%)$ \\
\hline Ransom et al. [11] & 1997 & U HMG** & 20 & $4(20 \%)$ \\
\hline Dale et al. [14] & 1999 & $\mathrm{U} \mathrm{FSH}^{* * *}$ & 19 & $5(26 \%)$ \\
\hline Guan et al. [12] & 2007 & $\mathrm{CC}^{*}$ & 67 & $9(13 \%)$ \\
\hline Katayama et al. [13] & 2010 & U HMG** & 16 & $11(69 \%)$ \\
\hline TOTAL & & & 208 & $44(21 \%)$ \\
\hline
\end{tabular}

$* \mathrm{CC}=$ Clomiphene Citrate, $* * \mathrm{U}$ HMG $=$ Urinary Human Menopausal Gonadotrophin, $* * * \mathrm{U}$ FSH $=$ Urinary Follicle Stimulating Hormone incubators or laminar flow hoods or other expensive instruments. A low clinical pregnancy rate should be expected for each attempt, but the cumulative pregnancy rate after 3-4 attempts should be similar to the pregnancy rate for a single IVF attempt. However, the fee difference between GUT and IVF is such that one may perform as many as approximately 20 GUT procedures for the cost of $1 \mathrm{IVF}$ procedure (Table 1). Therefore, poor people may afford GUT but not IVF. In addition, the low invasiveness of GUT makes it possible to repeat this procedure monthly or every other month in case of failure.

\section{GUT procedure}

\section{Stimulation of ovulation, timing the day of HCG administration and the day of GUT}

- Administer $150 \mathrm{mg}$ daily orally of Clomiphene citrate for 5 consecutive days, from day 3 to day 7 of the cycle.

- On day 7 or 8 do an ultrasound examination: if the endometrium is too thin (less than $6 \mathrm{~mm}$ ) administer human menopausal gonadotropin (HMG) 75 or 150 IU daily sc or im starting from day 8 .

- When the dominant follicle reaches a mean diameter of 17-18 mm administer human chorionic gonadotropin (HCG) $5000 \mathrm{IU}$ sc or im.

- If the patient has on average short cycles, you may need to administer HCG even earlier, since ovulation may occur pretty early, even with follicles with a diameter of $15-16 \mathrm{~mm}$. In fact, no down regulation is administred with GnRh agonists and also no antagonists are being administered. If ovulation actually takes place prematurely, fluid may be aspirated from the Douglas pouch and searched for COCs, or GUT may be converted to IUI if the tubes are patent. In case no oocytes are found due to premature ovulation, during the following attempt HCG should be administered 24 hours earlier.

\section{Preparation of the medium}

In the early days of IVF, patient's serum was added to the culture medium as a source of proteins in a percentage of 10 to $20 \%$ [15]. Below is depicted a simple method to obtain serum from the patient, which will be used as a medium for handling the gametes.

1. Draw $20 \mathrm{ml}$ of blood from the female patient 2.5 hours before GUT.

2. Transfer the blood into two $10-12 \mathrm{ml}$ empty sterile test-tubes, with screw caps, containing no anticoagulants or other chemicals.

3. Wait 30-35 minutes in order to obtain a good clotting.

4. Centrifuge both test tubes for 10 minutes at 2500-3000 rpm.

5. Aspirate all the supernatant, the serum, taking care not to aspirate the blood cells and transfer it to a clean, sterile test-tube.

6. Hermetically close this tube with its cap and plunge it into a water bath at $56-57^{\circ} \mathrm{C}$ for 30 minutes, paying attention that the water from the water bath does not enter into the test-tube. Heat denaturation of the serum will thus be achieved. If the tube cap does not close hermetically, place the test tube vertically in a test-tube rack inside the water bath taking care that the water from the bath does not enter into the tube.

7. Remove the test-tube from the water bath and keep it vertically at room temperature. Do not use the medium until it has cooled down to room temperature. 
8. 6-7 $\mathrm{ml}$ of serum will thus be at hand for both preparation of the sperm and handling of the COCs.

\section{Preparation of the sperm}

1. The husband should produce the sperm into a sterile plastic gobelet, 1.5 hours before the procedure.

2. Allow 30 minutes for liquefaction of the sperm.

3. Pour all the sperm into a sterile $10-12 \mathrm{ml}$ test tube.

4. Centrifuge the sperm for 10 minutes at $2500 \mathrm{rpm}$.

5. Remove all the supernatant leaving the sperm pellet at the bottom of the test tube (you may use an insulin syringe connected to a long needle).

6. Add $2 \mathrm{ml}$ of medium (the denaturated serum) into the test tube.

7. Shake the test tube in order to stir all the pellet and mix it with the medium.

8. Centrifuge the test tube for 5 minutes at $2000 \mathrm{rpm}$.

9. Remove all the supernatant and cover the pellet with $1 \mathrm{ml}$ of fresh medium.

10.Shake the test tube so that all the pellet will mix up with the medium.

11. Keep the test tube vertically in a test-tube rack, so that part of the debris and immotile sperm will sink to the bottom of the test-tube.

\section{Steps of the GUT procedure}

1. On the day of ovulation, the patient should be fasting and should empty the bladder just before the procedure. 36 hours after HCG insert a vaginal speculum and flush the vagina with saline through a $100 \mathrm{ml}$ syringe without needle. Rub the left, right, anterior and posterior vaginal fornix with a swab. Repeat again with another 100 $\mathrm{ml}$ saline. Remove all the saline and dry the vagina with a dry swab. Remove the speculum. Insert the vaginal ultrasound probe with attached the needle guide. Perform the follicle aspiration using a follicle aspiration needle kit. Vacuum may be applied either through a small electric aspirator or by hand with a $20 \mathrm{ml}$ syringe connected to the needle kit. Apply a low vacuum aspiration, otherwise you may damage the oocytes. There is no need of anaesthesia, since with Clomiphene the number of follicles is very low: usually not more than 2 or 3 . The pain is very modest and well tolerated, however one may administer a pain killer 15-30 minutes before the procedure.

2. The follicular fluid will be collected in sterile plastic 10-12 ml testtubes. Pour the follicular fluid from the test-tubes into sterile Petri dishes (since COCs tend to sink to the bottom of the test-tube, it is advisable to pour first the top half of the test-tube into the Petri dish; thereafter, shake the test-tube and immediately pour the remaining of the follicular fluid into the same or another Petri dish).

3. Put the Petri dish under the stereo-microscope and search for the COCs first with the naked eye and then with the stereo-microscope. Transfer the COCs with as little follicular fluid as possible into a large drop of clean medium in a clean Petri dish. Do not remove the cumulus. For transferring the COCs you may use an i.v. needle, after removal of the metallic cannula and using only the outer plastic cannula (this should have an inner diameter of approximately 1 $\mathrm{mm}$ ), connected to a sterile insulin syringe.

4. Shift all the retrieved COCs to a second clean drop of medium in the same Petri dish and repeat the procedure to a third clean drop of medium in the same Petri dish, in order to completely wash out all the posible blood cells.

5. In the same Petri dish transfer a drop of $0.3 \mathrm{ml}$ of washed sperm taken from the upper part of the sperm test-tube.

6. Aspirate into the transfer catheter from its tip, with the help of a needle free insulin syringe, $0.05 \mathrm{ml}$ of air, then the washed sperm and the oocytes with their cumulus - the total volume of oocytes and sperm should not be more than $0.15 \mathrm{ml}$-. A certain amount of serum in the transfer volume is helpful, since it may help buffer the acidic environment of the uterine cavity [16]. Use single transfer catheters - usually transfer of embryos is being performed with double catheters having an outer cannula and an inner very thin cannula containing the embryos; in GUT however, SINGLE transfer catheters should be used, since they are wider and are not harmful for the large COCs.

7. Insert a vaginal speculum and clean the cervical os with a sterile, dry swab.

8. Insert the catheter, negotiate the cervix and gently reach the fundus of the uterus. If the catheter has no marks, it is wiser to preliminarly mark the catheter at $7 \mathrm{~cm}$ from the tip with a marker. Inject all. The small quantity of air preliminarly aspirated into the catheter will help to completely empty the catheter. Remove gently the catheter and the speculum. The patient may resume her normal activities a few minutes later.

9. It is advisable to administer oral antibiotics to the patient starting the day before GUT and continuing for another 4-5 days after GUT.

\section{Complications}

The complication rate of IVF is very low [17]. Complications may be both surgical and medical.

\section{Surgical complications}

1. Bleeding from the vaginal fornix: it is usually controlled with strong pressure with a thick swab. Rarely is it necessary to put stitches.

2. Bleeding from the ovary: some slight bleeding occurs from the punctured ovarian follicles, but it stops almost immediately: it is recommended to test preliminarly the coagulation situation of the patient.

3. It is important to avoid puncturing by mistake arteries or veins ( the internal iliac vessels are pretty close, but are normally easy to locate): if a vessel is punctured by mistake the aspirated fluid will be pure blood. In this case, do not move the vaginal probe, gently withdraw the needle and strongly press with the probe against the punctured site for a few minutes.

4. Avoid puncturing other nearby organs, such as the bowel, which is easy to identify by ultrasound.

5. The follicles to puncture should be very close to the vaginal fornix: if they are far, the risk of injuring other organs increases.

6. To avoid injuring the bladder, ask the patient to empty her bladder just before the oocyte pick up procedure.

\section{Medical complications}

1. Infections may occur as a result of a vaginitis, or an unsufficient washing and cleansing of the vagina with saline before OPU. Therefore, in case of vaginitis, postpone the procedure and first treat the vaginitis. 
2. Also avoid puncturing an hydro-salpinx. The ovarian hyperstimulation syndrome does occur very rarely when using Clomiphene for ovulation stimulation.

\section{Expected pregnancy rates}

A pregnancy rate of $14-15 \%$ is expected for each GUT procedure [13]. However, since what has just been depicted is a simplified procedure tailored for gynecologists who do not have a specific training in ART, one should predict lower results at the beginning, due to a learning curve. Therefore, if we anticipate a halving of this pregnancy rate, at least at the beginning, the cumulative pregnancy rate after 4 attempts should be approximately similar to a single IVF procedure (29\%) [18]. After some time, however, the pregnancy rate of GUT may gradually increase, because of the gain of familiarity with the procedure by the gynecologists. However, some gynecologists already perform IUIs in their offices: for these doctors the learning curve of GUT could be rather rapid, since they are already familiar with ovulation stimulation, ultrasound monitoring and sperm preparation.

\section{Instruments, plastic ware, medicines and costs}

One of the main features of this procedure is the reduction of costs, hence the inclusion of a detailed analysis of the costs.

1. A benchtop centrifuge (150 USDollars); an ultrasound machine with a vaginal probe and a needle guide $(1,800-2,300$ USD); a stereo microscope with a led bulb from below (250 USD); possibly a phase contrast microscope for the sperm (1,000 USD). Total cost of instruments: approximately 4,000 USD, including freight expenses.

2. A plastic sterile gobelet for sperm collection; 7-8 sterile, plastic 10$12 \mathrm{ml}$ test tubes, 3-4 plastic, sterile $50 \mathrm{~mm}$ diameter Petri dishes, 2-3 plastic insulin syringes, one sterile needle kit for follicular aspiration, one plastic sterile transfer catheter: the cost of all these disposable items should be around 25 USD for each procedure.

3. Clomiphene citrate $50 \mathrm{mg}, 15$ tablets: 2 USD; HCG 5,000 IU, one vial: 1.5 USD; oral antibiotics for 5 days: 5 USD; a pain killer, one tablet: 0.5 USD $=9$ USD. In case HMG is added one should add another 20-30 USD.

The total fee of a GUT procedure could be: 4 times a gynaecological consultation (X) - if, for example, a gynecological consultation fee is 15 USD -, the doctor's fee for the GUT could be: $4 \times 15=60$ USD + depreciation of instruments: 8 USD + drugs: 9 USD + disposable items: 25 USD, totalling: 102 USD. With HMG the cost would rise to 125-130 USD.

Prices are very different for an IVF in an infertility centre: 2,000 to 3,000 USD compared to around 100/130 USD for a GUT procedure. Even though the pregnancy rate is obviously higher with IVF than GUT, the difference in costs is huge: with the cost of one IVF procedure, a patient may perform as many as 20 or even more GUT procedures.

\section{Discussion}

Overcoming tubal infertility by surgically inserting part of an ovary into a cornual part of the uterus has been successfully attempted in the past [19]. In 1978 the first IVF baby was born [20], showing that even in humans, fertilization and early cleavage may take place outside the body, in a completely artificial environment, a Petri dish within an artificial medium. More recently several authors [6-14] have transferred directly oocytes and sperm into the uterus soon after oocyte pick up (GUT), showing that in humans, fertilisation and early cleavage may occur in the uterus, albeit the fallopian tube is the ideal site of fertilization and early cleavage. An average pregnancy rates of $20 \%$ has been reported for $\mathrm{GUT}^{1}$ (Table 1). Even if the actual pregnancy rate drops to half at the beginning of the learning curve for an inexperienced doctor, the cumulative pregnancy rate after approximately 4 attempts would be similar to a single IVF attempt. Even during the early days of IVF, results were discouragingly low and only increased significantly in the last 20 years, due to an enormous world wide research in the field. In the mid eighties, a valid alternative to IVF, namely laparoscopic gamete intra fallopian transfer (GIFT), showed a pregnancy rate remarkably higher than IVF [21], but it became rapidly obsolete since after a few years, the much simpler transvaginal ultrasound guided oocyte pick up became common place and also because GIFT had the limitation that it could not be used for tubal infertility. It is tempting to speculate therefore that with time and adequate research even GUT may improve its results.

Contrarily to what is generally thought, treating infertility with simplified new technologies is not that difficult. Treating infertility with simple techniques by gynecologists or even general practitioners is a must if we actually wish to implement what is generally accepted worldwide, that everyone has the right to reproduce, whether rich or poor and we cannot expect that expensive infertility centres may accomplish this mandate.

In our view, infertility treatment should stop being considered a top specialty and should return to be practised by gynaecologists. For poor people, only in rare cases, for example with severe male infertility where ICSI is needed, should it be necessary to resort to specialised centres. GUT may be performed in an office setting, in only a few minutes, with very few basic instruments and with limited training. Concerning the medium for handling oocytes and sperm, the use of the patient's serum is suggested, since it has a rather stable physiological $\mathrm{pH}$ and osmolarity; and in any case the patient's serum will not be used as a proper culture medium, but only as a washing and handling medium, since oocytes will stay outside of the body for only a few minutes. Commercial media are pretty expensive, they expire after a short time and the cold chain is hard to comply with in developing countries. The doctor may perform all the steps of the procedure either alone or with the help of a nurse. Doctors should also become familiar with the examination of the sperm with a phase contrast microscope, although it is not absolutely necessary, since sperm tests are carried out in general labs. Involving gynaecologists in simple infertility treatments such as IUI and GUT may also help in spreading infertility prevention advice and wisdom among the general population, since these doctors see patients even when they are not yet infertile, while infertility centres mostly see patients already and permanently infertile.

The costs and fees for the patients have been calculated by taking into consideration the costs of the instruments and disposables as they appear in the internet (companies selling online, such as Alibaba, Indiamart, ebay and so on) and by calculating the time and the commitment of the doctors in performing the above techniques and relating them to their normal consultation fees. Depreciation of the instruments is calculated in 10 years and the cost of depreciation is shared among 500 cases, which means: an average of one procedure each week for 10 years. However, the microscopes and the centrifuge may last a life time if properly handled. On the contrary, the ultrasound machine may last less because of the electricity fluctuations in developing countries. It is advisable to protect the electronics of this instrument with an electric surge protector or other such devices. However, the depreciation allowance of the ultrasound machine may 
be shared with other users of this instrument in normal gynecology or obstetrics. In addition, doctors performing GUT may also perform IUI which needs the same instruments. Therefore depreciation allowance may be shared by many more patients. A total fee for the patient of little more than 100 USD has been estimated.

Lowering costs for the poor should start from the very beginning. A careful history should be taken and a clever evaluation of each case should be made.

For example, in case of a woman of $40-42$ years of age or more, she should not be encouraged to undergo any treatment and she should be told honestly that her chances of becoming pregnant are very low, even with sophisticated procedures.

One should limit preliminary examinations and tests to a minimum in order to reduce costs here too: in most cases, a sperm test, a hysterosalpingography and hormonal evaluations in case of menstrual irregularities of the female partner are enough.

However good medical practice should not be ignored even when low cost reproductive procedures are being implemented, such as preliminary checking for general health of the patients, checking for infective diseases, such as HIV, Hepatitis, Syphilis and also for local infections such as urethritis and vaginitis. It was decided to use Clomiphene, since it is a cheap oral drug, it has been used by the teams which developped GUT, it exceptionally gives rise to hyperstimulation syndromes or twins, which may burden economically and medically the patient, it produces few eggs allowing for oocyte pick-up with no anesthesia or with just the administration of a pain killer and it is not less effective in terms of results than IUI, when compared to gonadotropins [22]. In addition, the fewer eggs collected with clomiphene ovarian stimulation may have a lower rate of aneuploidy [23] as compared to gonadotropins and therefore the take home baby rate may be quite satisfactory as compared to the greater loss of embryos/foetuses after gonadotropin stimulation. Sometimes, however clomiphene may produce a thin endometrium: in these cases a small dose of HMG may be added daily starting from day 8 until HCG administration.

In the end, GUT may even be employed by infertility centres as they do with IUI: a preliminary low cost GUT procedure to be attempted a couple of times, before IVF in patients with occluded tubes.

\section{Conflict of interest}

The authors report no conflict of interest.

\section{Funding}

The authors report no funding whatsoever.

\section{Contribution of authors}

Conception of the study: LF, RA, RK, SS and GB

Literature search: MA, RR, NA and HR

Preparation of the manuscript: LF

\section{Acknowledgments}

The authors thank the staff of CEFERMAD (Centre de Fertilité de Madagascar), namely, Nathalie Razaiarisoa.

\section{References}

1. Inhorn MC and Patrizio P (2015) Infertility around the globe: new thinking on gender, reproductive technologies and global movements in the 21st century. Hum Reprod Update 21: 411-426. [Crossref]
2. Nsa AM and Formigli L (2012) Low cost, simple, intrauterine insemination procedure with unwashed centrifuged husband's sperm for developing countries. Afr J Reprod Health 16: 175-179. [Crossref]

3. Klerckx E. IVF for 200 euro per cycle: first real-life proof of principle that IVF is feasible and effective for developing countries. European Society of Human Reproduction and Embryology 2013; available from: http://www.eshre.eu/London2013/Media/Releases/ Elke-Klerckx.aspx

4. Van Blerkom J, Ombelet W and Klerkx E (2014) First births with a simplified culture system for clinical IVF and embryo transfer. Reprod Biomed Online 28: 310-320. [Crossref]

5. Taymor ML, Ranoux CJ and Gross GL (1992) Natural oocyte retrieval with intravaginal fertilisation: a simplified approach to in vitro fertilisation. Obstet Gynecol 80: 888-891. [Crossref]

6. Craft I and McLeod F (1982) Human pregnancy following oocyte and sperm transfer to the uterus. Lancet 319: 1031-1033. [Crossref]

7. Veersema S, Bernardus RE, Veersema D, Vermeiden JPW, Schoemaker J (1989) Pregnancy following transcervical transfer of oocyte and sperm. $N$ Engl J Med 320: 1499-1452. [Crossref]

8. Kubik CJ, Guzick DS, Berga SL, Zeleznik AJ (1990) Establishment of pregnancies in humans after transcervical transfer of gametes immediately after oocyte retrieval. Fertil Steril 54: 1174-1176. [Crossref]

9. Rizk B, Bekir JS, Avery S, Smith S, Edwards RG (1990) Intrauterine replacement of oocytes with bound sperms: a pilot study. Hum Reprod 5 (suppl. 1): 5.

10. Sterzik K, Rosenbusch B, Grab D, Lauritzen C (1991) Pregnancies following direct oocyte-sperm transfer (DOST): a simple alternative to conventional in vitro fertilization (IVF). J In Vitro Fert Embryo Transf 8: 241-242. [Crossref]

11. Ransom MX, Garcia AJ, Doherty K, Shelden R, Kemmann E (1997) Direct gamete uterine transfer in patients with tubal absence or occlusion. $J$ Assist Reprod Genet 14 35-38. [Crossref]

12. Guan Q, Zhang Q, Sun W (2007) Gametes intrauterine transfer versus in vitro fertilization for poor responders. Int J Gynecol Obstet 96: 37-38. [Crossref]

13. Katayama KP, Jambor V, Milosavljevic Sue, Vanschaick M, Katayama A (2010) Uterine sperm-egg transfer: a cost-effective alternative to IVF? RBMO 20: 764-767. [Crossref]

14. Dale B, Fiorentino A, De Stefano R, Di Matteo L, De Simone ML, et al. (1999) Pregnancies after activated oocyte transfer: a new option for infertility treatment. Hum Reprod 14: 1771-1779. [Crossref]

15. Benadiva CA, Kuczynski-Brown B, Maguire TG, Mastroianni L Jr, Flickinger GL (1989) Bovine serum albumin (BSA) can replace patient serum as a protein source in an in vitro fertilization (IVF) program. J In Vitro Fert Embryo Transf 6: 164-167. [Crossref]

16. Dale B, Menezo Y, Cohen J, Di Matteo L, Wilding M (1998) Intracellular pH regulation in the human oocyte. Hum Reprod 13: 964-970. [Crossref]

17. Seyhan A, Ata B, Son W, Dahan M, Tan SL (2014) Comparison of complication rates and pain scores after transvaginal ultrasound guided oocyte pickup procedures for in vitro maturation and in vitro fertilization cycles. Fertil Steril 101: 705-709. [Crossref]

18. Dyer SJ, Kruger TF (2012) Assisted reproductive technology in South Africa: First results generated from the South African register of assisted reproductive techniques. SAMJ 102: 167-170. [Crossref]

19. Estes WL Sr (1909) A method of implanting ovarian tissue in oder to maintain ovarian function. Pa Med J 13: 610-615.

20. Steptoe PC, Edwards RG (1978) Birth after the reimplantation of a human embryo. Lancet 12: 366. [Crossref]

21. Formigli L, Coglitore MT, Roccio C, Belotti G, Stangalini A, et al. (1990) One-undredand-six gamete intra-Fallopian transfer procedures with donor semen. Hum Reprod 5 : 549-552. [Crossref]

22. The ESHRE Capri Workshop Group (2009) Intrauterine insemination. Hum Reprod Update 15: 265-277.

23. Allahbadia GN (2016) Stimulation and aneuploidy: why are milder stimulation protocols better? IVFLITE 3: 41-45

Copyright: (C2019 Formigli L. This is an open-access article distributed under the terms of the Creative Commons Attribution License, which permits unrestricted use, distribution, and reproduction in any medium, provided the original author and source are credited. 